\title{
UHECR mass composition from anisotropy of their arrival directions with the Telescope Array SD
}

\author{
Mikhail Kuznetsov ${ }^{a, b, *}$ and Peter Tinyakov ${ }^{a, b}$ on behalf of the Telescope Array \\ Collaboration \\ (a complete list of authors can be found at the end of the proceedings) \\ ${ }^{a}$ Service de Physique Theorique, Universite Libre de Bruxelles, Boulevard du Triomphe CP225, 1050 \\ Brussels, Belgium \\ ${ }^{b}$ Institute for Nuclear Research of the Russian Academy of Sciences, 60th October Anniversary Prospect \\ 7a, 117312 Moscow, Russia \\ E-mail: mkuzn@inr.ac.ru
}

We propose a new method for the estimation of ultra-high energy cosmic ray (UHECR) mass composition from a distribution of their arrival directions. The method employs a test statistic (TS) based on a characteristic deflection of UHECR events with respect to the distribution of luminous matter in the local Universe modeled with a flux-weighed 2MRS catalog. Making realistic simulations of the mock UHECR sets, we show that this TS is robust to the presence of galactic and non-extreme extra-galactic magnetic fields and sensitive to the mass composition of events in a set. We apply the method to Telescope Array surface detector data for 11 years and derive new independent constraints on fraction of protons and iron in p-Fe mix at $E>10 \mathrm{EeV}$. At $10<E<100 \mathrm{EeV}$ the data favors increase of allowed proton fraction and decrease of allowed iron fraction, while at $E>100 \mathrm{EeV}$ - pure iron or even more massive composition. This result is in tension with Auger composition model inferred from spectrum- $X_{\max }$ fit at $2.7 \sigma(2.0 \sigma)$ for PT'11 (JF'12) regular GMF model.

$37^{\text {th }}$ International Cosmic Ray Conference (ICRC 2021)

July 12 th - 23rd, 2021

Online - Berlin, Germany

\footnotetext{
*Presenter
} 


\section{Introduction}

Despite the experimental progress in detection of ultra-high energy cosmic rays (UHECR) and growing quality and quantity of data, our understanding of this phenomenon is hampered by three coupled unsolved problems: UHECR sources, nature of UHECR particles and cosmic magnetic fields.

While the arrival directions of UHECR are reconstructed with the precision of $1-1.5^{\circ}$ and no systematic errors, the directions to the UHECR sources cannot be determined with any precision because of deflection in cosmic magnetic fields by potentially much larger angles. These deflections are uncertain because of both the unknown particle charge and uncertainties in the magnetic fields. The determination of the primary particles charge is prone to uncertainties of hadronic interaction models. The existing measurements [1-4] have large errors and may contain unknown systematic effects. Cosmic magnetic fields are also not known sufficiently well. Experimentally, only loose bounds exist on the extragalactic fields [5, 6]. A rough magnitude of the coherent Galactic magnetic field (GMF) is known to be several $\mu G$ [7]. However, its general structure is unknown. Several proposed phenomenological models $[8,9]$ should be considered as examples of what the field might be, at best.

Our approach is to reduce three uncertainties - sources, composition and magnetic fields by additional assumptions. A most robust assumption can be made about the source distribution in space: in all existing models they follow the matter distribution. If one assumes in addition that the sources are sufficiently numerous to be treated on statistical basis, the uncertainty related to sources is essentially eliminated. We will refer to this source distribution as Large-scale Structure (LSS) source model.

To disentangle the uncertainties of magnetic field from that of mass composition it is instructive to note that the former is estimated to be a factor $\sim 2$ in terms of average deflections, if the EGMF is not extremely strong or coherent (see Ref. [10] for detailed discussion). While the uncertainty due to composition would be as large as factor 26 if we try to distinguish protons from iron nuclei. This simple relation gives the opportunity to study and constrain mass composition as the most uncertain parameter of the UHECR flux. The study is made by comparing observed distribution of UHECR in the sky with those simulated for different UHECR compositions.

The comparison is made using a single characteristic parameter - an average deflection from the LSS parameterized as a width of the Gaussian spread of a point source, average over UHECR events is a set. While the contribution of complicated coherent galactic magnetic field to deflections is likely large, we have shown that this observable is mostly insensitive to GMF details but still sensitive to the overall magnitude of deflections [10]. At the same time this observable have a discriminating power with respect to different compositions of UHECR in an event set.

In the present note we apply the developed method to the recent TA SD data and derive the constraints on the composition of UHECR with $E>10 \mathrm{EeV}$.

\section{The experiment and the datasets}

Telescope Array [11,12] is the largest cosmic-ray experiment in the Northern Hemisphere. It is located at $39.3^{\circ} \mathrm{N}, 112.9^{\circ} \mathrm{W}$ in Utah, USA. The observatory includes a surface detector array 

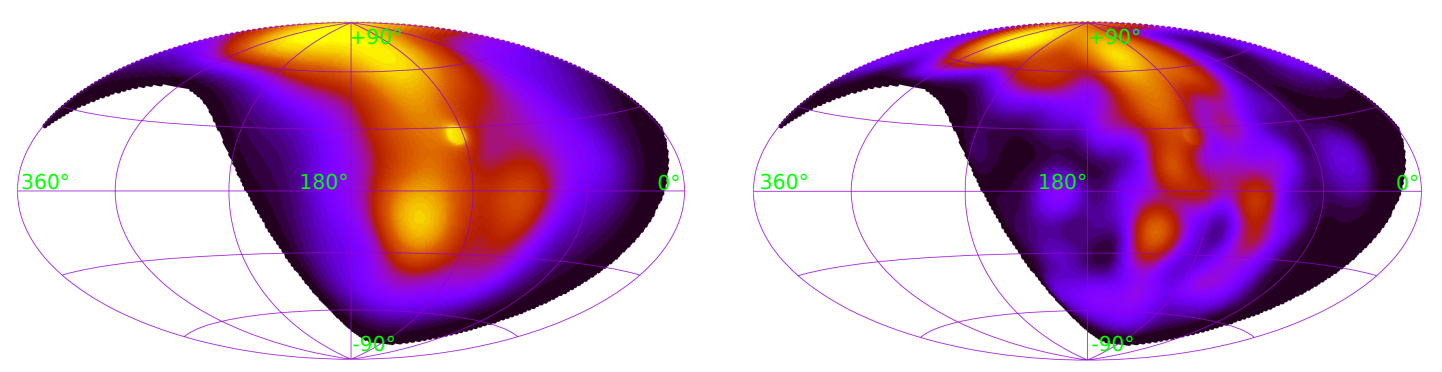

Figure 1: Examples of UHECR flux model maps $\Phi_{k}$ for TS calculation. Proton attenuation length and uniform smearing of the sources is assumed: $\theta(100 \mathrm{EeV})=10^{\circ}$; energies are $E_{k}=57 \mathrm{EeV}$ (left) and $E_{k}=100 \mathrm{EeV}$ (right). Maps are shown in galactic coordinates for TA SD field of view.

(SD) and 38 fluorescence telescopes grouped into three stations. The SD consists of 507 stations that contain plastic scintillators each of $3 \mathrm{~m}^{2}$ area (SD stations). The stations are placed in the square grid with the $1.2 \mathrm{~km}$ spacing and covers the area of $\sim 700 \mathrm{~km}^{2}$. The TA SD is capable of detecting EAS in the atmosphere caused by cosmic particles of EeV and higher energies. The TA SD operates since May 2008.

We use the 11 years of TA SD data (from 11.05.2008 to 10.05.2019) in this study and employ the TA SD set with cuts previously set for anisotropy studies [13]. Namely: (1) each event includes at least 5 SD counters; (2) $\chi^{2} /$ d.o.f. doesn't exceed 4 for both the geometry and the LDF fits; (3) arrival direction is reconstructed with accuracy less than $5^{\circ}$; (4) fractional uncertainty of $S_{800}$ is less than $25 \%$; (5) largest signal counter is surrounded by 4 working counters that are not necessarily its nearest neighbors; (6) reconstructed zenith angle of event is less than $55^{\circ}$; (7) reconstructed energy is greater than $10 \mathrm{EeV}$.

By construction our method is highly sensitive to outlier events (events that are far from any LSS-source). Therefore to stay on a safe side we apply an additional cut that removes all the events coinciding with lightnings in TA SD detector. We cut-off events temporarily matching with NLDN lightnings [14]; this reduce the number of TA SD events only by $\sim 0.7 \%$ [15]. The final data set contains 4769 events with $E>10 \mathrm{EeV}, 127$ events with $E>57 \mathrm{EeV}$ and 15 events with $E>100 \mathrm{EeV}$.

\section{The analysis}

The UHECR sources, regardless of their nature, are expected to trace the matter distribution. In the limit when the density of sources is sufficiently high so that they can be treated statistically, the expected UHECR flux can be calculated, as a function of energy, with essentially one free parameter, the typical deflection angle $\theta$ which encodes uncertainties and unknowns of Galactic and extragalactic magnetic fields and of chemical composition.

The mass distribution in the Universe was inferred from the 2MASS Galaxy Redshift Catalog (XSCz) that is derived from the 2MASS Extended Source Catalog (XSC). We use the sample corrected for catalog incompleteness and cut-off sources that are closer than $5 \mathrm{Mpc}$ to us. We have assumed that sources follow the matter distribution, and propagated UHECRs from sources 

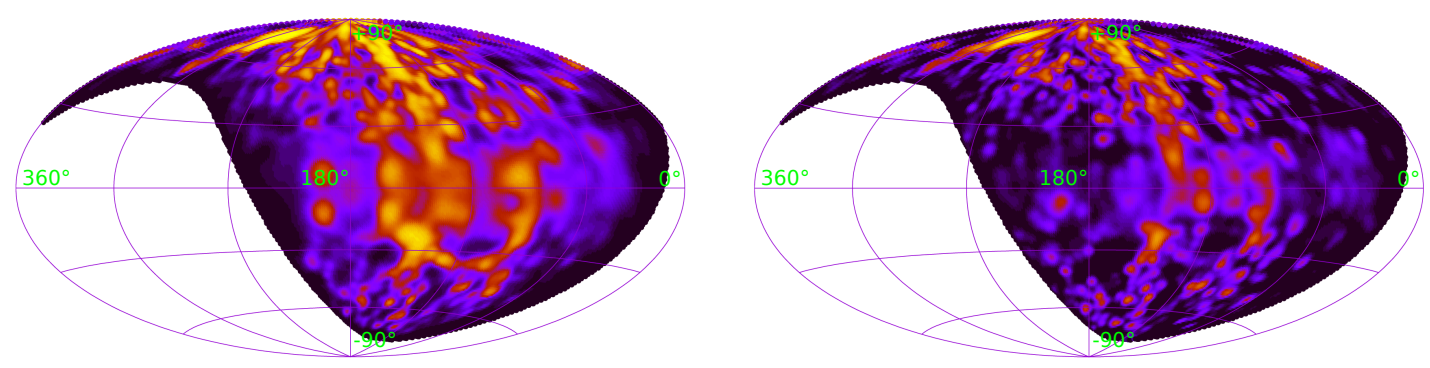

Figure 2: Examples of UHECR flux model maps $F_{k}$ for mock UHECR sets simulation. Maps for protons with b-dependent smearing of the sources and PT'11 regular GMF: $E=57 \mathrm{EeV}$ (left) and $E=100 \mathrm{EeV}$ (right).

to the Earth taking full account of the energy attenuation processes under the assumption that the primary particles are protons with $E^{-2.5}$ injection spectrum. Accounting for the energy dependence does not introduce additional parameters as the deflection angles are inversely proportional to event energies and can be expressed in terms of a single parameter $\theta_{100}$ - the deflection at a reference energy $E_{0}=100 \mathrm{EeV}$. We bin the energies in log-uniform intervals with lower boundaries $E_{k}$ (ten bins per energy decade with the highest bin an open interval $E>180 \mathrm{EeV}$ ) and neglect the energy dependence within each bin. The arrival directions then are with the spherical Gaussian function (Fisher distribution) with the opening angle $\theta=\theta\left(E, \theta_{100}\right)$ containing $\sim 63 \%$ of probability.

For a given smearing parameter $\theta_{100}$ and given energy bin we construct the sky map of the expected flux making use of the source distribution in space and the exposure of the experiment. We normalize a flux map $\Phi_{k}\left(\theta_{100}, \mathbf{n}\right)$ obtained this way to a unit integral over the sphere so that it can be interpreted as a probability density to observe an event from the direction $\mathbf{n}$. Two examples of such maps are shown in Fig. 1. Finally, we define our test statistics $T S\left(\theta_{100}\right)$ as follows:

$$
T S\left(\theta_{100}\right)=-2 \sum_{k}\left(\sum_{i} \ln \frac{\Phi_{k}\left(\theta_{100}, \mathbf{n}_{i}\right)}{\Phi_{\text {iso }}\left(\mathbf{n}_{i}\right)}\right),
$$

where the internal sum runs over the events observed in the energy bin $k$ and the normalization factor $\Phi_{\text {iso }}\left(\mathbf{n}_{i}\right)$ corresponds to the isotropic distribution of sources - a uniform flux modulated by the exposure function. In the limit of a large number of events, this test statistics is distributed around its minimum according to $\chi^{2}$-distribution with one degree of freedom.

We now want to test its behavior for different compositions and magnetic field models, and therefore we need to generate Monte-Carlo event sets that follow these models. To this end we simulate another set of flux maps that is used to generate model event sets: we use the same sources distribution but attenuate the flux according to SimProp [16] fits for each particular primary [17], apply a latitude-dependent smearing due to random GMF and additionally process the flux through the regular GMF. To avoid confusion, we denote these maps as $F_{k}$. Two examples of these maps are shown in Fig. 2. Both $\Phi_{k}$ and $F_{k}$ maps are modulated by the exposure function of the TA experiment, for which we take the geometrical exposure. The procedure described in more detail in Ref. [10]. Finally, we use the maps $F_{k}$ to generate the test event sets which we need to study the behavior of the test statistics Eq. (1). The mock event sets are generated by throwing random events 


\begin{tabular}{|c|c|c|c|c|}
\hline $\lg (E / \mathrm{EeV})$ & $f_{\mathrm{p}}^{\mathrm{UL}}$, GMF PT'11 & $f_{\mathrm{p}}^{\mathrm{UL}}, \mathrm{GMF} J F^{\prime} 12$ & $f_{\mathrm{Fe}}^{\mathrm{UL}}, \mathrm{GMF}$ PT'11 & $f_{\mathrm{Fe}}^{\mathrm{UL}}, \mathrm{GMF} \mathrm{JF}{ }^{\prime} 12$ \\
\hline $1.00-1.25$ & 0.44 & 0.41 & 0.89 & 0.91 \\
\hline $1.25-1.50$ & 0.66 & 0.64 & 0.64 & 0.70 \\
\hline $1.50-1.75$ & 0.81 & 0.83 & 0.36 & 0.35 \\
\hline $1.75-2.00$ & 0.93 & 0.94 & 0.11 & 0.12 \\
\hline$>2.00$ & 0.00 & 0.00 & 1.00 & 1.00 \\
\hline
\end{tabular}

Table 1: The $68 \%$ C.L. constraints on the fractions of protons and fraction of iron in $p-F e$ mix derived separately for two regular GMF models (see text for details).

with the TA SD spectrum [18] and accepting them with the probability given by the corresponding flux map $F_{k}$ at the position of the event.

As it was shown in Ref. [10] the likelihood shape is robust to the presence of regular GMF and non-extreme EGMF and even to reasonable magnetic field magnitude and model variation. It opens a way for a new method of UHECR mass composition estimation. The position of the TS minimum, $\theta_{100, \min }$, is a proxy of the primary particles deflection from their sources that is directly related to the charge of these particles. Therefore, by measuring $\theta_{100, \min }$ in the data one could estimate the mean charge of UHCER in a given sample. This estimation can be made by comparing the value of $\theta_{100, \min }$ for the data with the distribution of $\theta_{100, \min }$ in mock event sets of a particular UHECR composition model. Since we only have one number $\theta_{100, \min }$ determined from the data, the exact composition is impossible to determine because the same value of $\theta_{100 \text {,min }}$ may correspond to different composition models. Nevertheless, the composition can be constrained by excluding models where the measured value of $\theta_{100, \text { min }}$ never occurs or occurs rarely.

\section{Results}

In this study we limit ourselves with a simplified approach where the UHECR consist of a 2 component mixture with one light and one heavy component. We use this approach mostly due to its simplicity and left the multicomponent analysis for the future work. Despite the simplification, the results of this approach may still be of practical importance. For instance, for the proton-iron mix the upper-bound on the proton fraction derived in this setup is conservative in the sense that it will hold if iron is replaced by lighter species. The same applies to the upper-bound on the fraction of iron. First, we constrain the constant proton fraction $f_{\mathrm{p}}$ and iron fraction $f_{\mathrm{Fe}}$ in separate energy bins. We set the log-uniform bins of 0.25-decade width starting at $E=10 \mathrm{EeV}$. The $68 \%$ C.L. constraints are shown in Table 1 and plotted in Fig. 3 together with TS shapes for data. We derive these constraints separately for regular GMF models of Ref. [8] and Ref. [9]. As one can see the results for different GMF models does not differ much.

Next, we use our approach to constrain the Auger composition model derived from spectrum and $X_{\max }$ fit [3]. We set the interval $E>57 \mathrm{EeV}$ and interpret the Auger best-fit model as the injection of pure silicon nuclei with cutoff, according to Ref. [17]. We employ the silicon attenuation functions for mock set generation from the same Ref. [17] and refer to this scenario as Auger-like model. The resulting $\theta_{100 \text {,min }}$ distributions are shown in Fig. 4 together with the $\theta_{100 \text {,data }}$ point for 

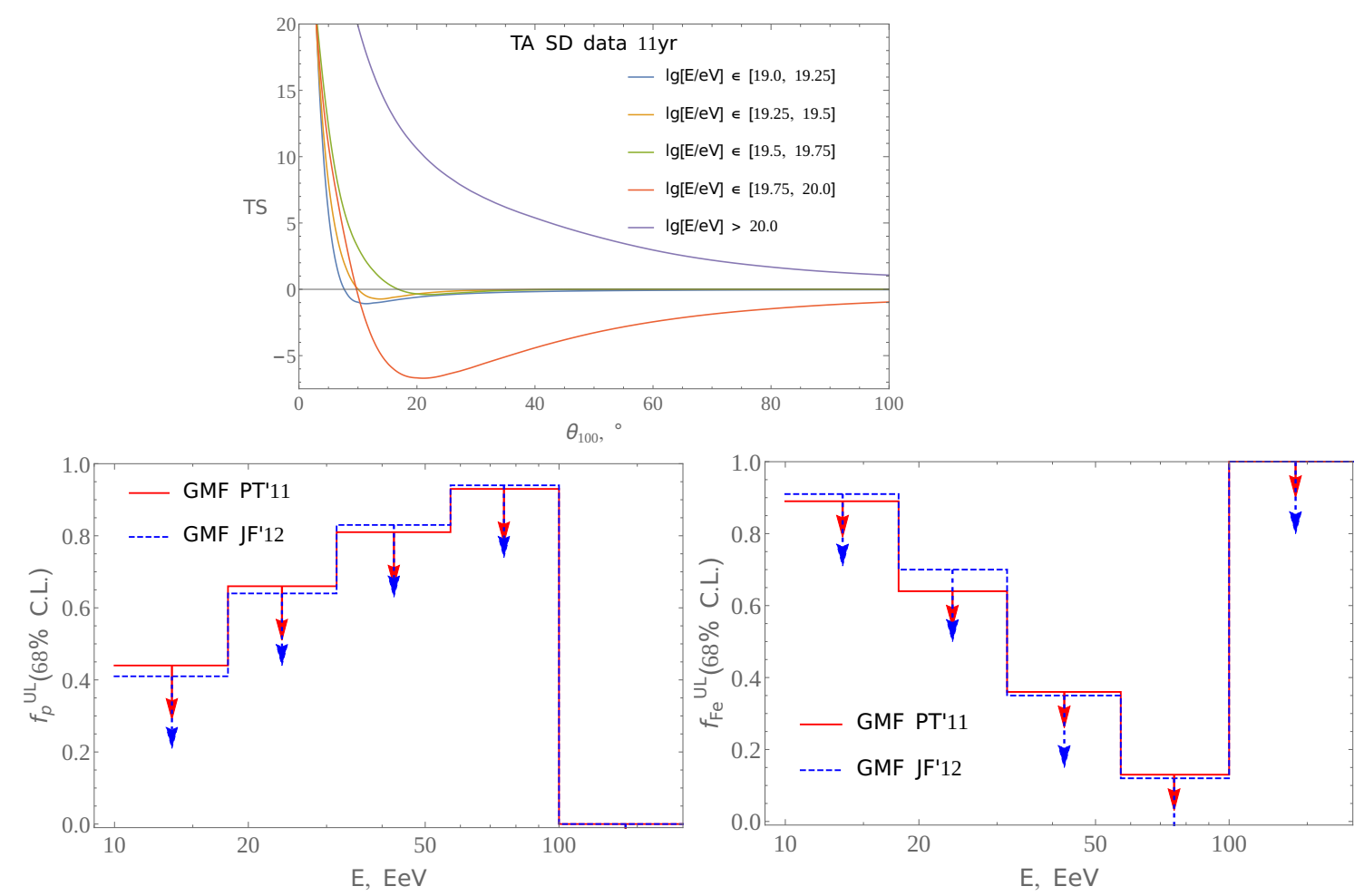

Figure 3: Top: Test statistic computed for TA SD data in separate energy bins. Bottom: constraints on fraction of protons (left) and iron (right) in p-Fe mix at 68\% C.L. derived from TS of data for regular GMF models of PT'11 and JF'12.

the same energy interval. The TS for the TA data does not have minimum thus favoring very heavy composition. We derive the p-value to have such $\theta_{100, \min }$ value in the Auger-like model for two models of regular GMF. These p-values are 0.0061 and 0.043 for the PT'11 and JF' 12 GMF models respectively. Note that we did not assume any energy rescaling between TA and Auger energy scales in this test.

\section{Conclusion}

We proposed a quantitative method to assess composition of UHECR by using information on their arrival directions and energies, under the assumption that sources follow the large-scale matter distribution in the Universe. The key point of the proposal is calculation of the typical deflection angle with respect to the LSS source model. This angle is defined as a minimum $\theta_{100, \min }$ of the likelihood function $T S\left(\theta_{100}\right)$, Eq. (1). It should be calculated for the data and compared to the same quantity calculated for the composition model in question. Our method has several advantages: it is based exclusively on measured UHECR arrival directions and energies of events which are most reliably determined from the reconstruction of air showers; it is not sensitive to the details of the regular GMF and to the presence of the non-extreme EGMF; it can give conclusive results even at highest energies where use of other methods of composition study is limited by low UHECR statistics. 


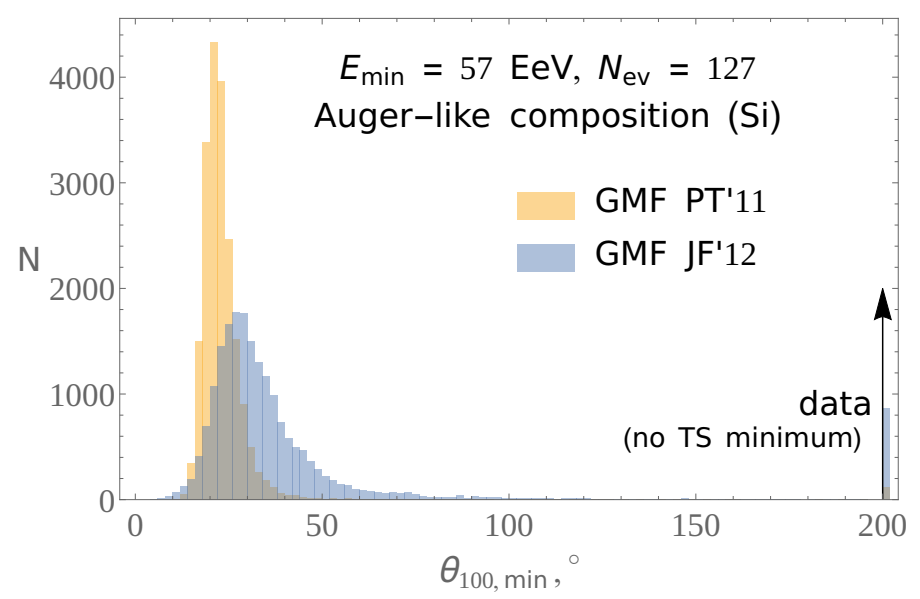

Figure 4: The distribution of $\theta_{100, \text { min }}$ for Auger-like composition model for regular GMF models of PT'11 and JF' 12 compared with the $\theta_{100, \text { data. }}$.

We applied the method to the 11 years of the TA SD data and derived the upper limits on the fraction of protons and iron in p-Fe mix composition. The results in separate energy bins show the monotonic decrease of the allowed iron fraction and increase of the allowed proton fraction for energies from $10 \mathrm{EeV}$ to $100 \mathrm{EeV}$, so that for $57<E<100 \mathrm{EeV}$ the data allows only a small heavy admixture. At $E>100 \mathrm{EeV}$ the situation changes completely and the data favors a pure iron composition ${ }^{1}$ and disfavors any proton admixture. We left the interpretation of these interesting results for the future works. It is also remarkable that the results are robust to the change of assumed regular GMF model.

We also employed our method to test the compatibility of TA data with composition model inferred by Auger. We used the Auger best-fit composition of Ref. [3] interpreted as the injected pure silicon according to Ref. [17] and tested it with the TA data. At $E>57 \mathrm{EeV}$ we found that the model is in tension with the TA data at the level of $2.7 \sigma(2.0 \sigma)$ for PT'11 (JF' 12) regular GMF model. It is worth noting that this discrepancy is dominated by the TA "ultra-heavy" events at $E>100 \mathrm{EeV}$ for that the injected silicon is not heavy enough.

Acknowledgments: The Telescope Array experiment is supported by the Japan Society for the Promotion of Science(JSPS) through Grants-in-Aid for Priority Area 431, for Specially Promoted Research JP21000002, for Scientific Research (S) JP19104006, for Specially Promoted Research JP15H05693, for Scientific Research (S) JP15H05741, for Science Research (A) JP18H03705, for Young Scientists (A) JPH26707011, and for Fostering Joint International Research (B) JP19KK0074, by the joint research program of the Institute for Cosmic Ray Research (ICRR), The University of Tokyo; by the Pioneering Program of RIKEN for Matter in the Universe (r-EMU); by the U.S. National Science Foundation awards PHY-1404495, PHY-1404502, PHY-1607727, PHY-1712517, and PHY-1806797; by the National Research Foundation of Korea (2017K1A4A3015188, 2020R1A2C1008230, \& 2020R1A2C2102800) ; IISN project No. 4.4501.18, and Belgian Science Policy under IUAP VII/37 (ULB). The application of the analysis method to data was supported by the Russian Science Foundation grant No. 17-72-20291 (INR). The foundations of Dr. Ezekiel R. and Edna Wattis Dumke, Willard L. Eccles, and George S. and Dolores Doré Eccles all helped with generous donations.

\footnotetext{
${ }^{1}$ Actually at $E>100 \mathrm{EeV}$, the data favors the deflections even larger than the pure iron nuclei should have. To infer the implied average mass we need to simulate the mock event sets with primaries beyond iron nuclei; we left this for future studies.
} 
The State of Utah supported the project through its Economic Development Board, and the University of Utah through the Office of the Vice President for Research. The experimental site became available through the cooperation of the Utah School and Institutional Trust Lands Administration (SITLA), U.S. Bureau of Land Management (BLM), and the U.S. Air Force. We appreciate the assistance of the State of Utah and Fillmore offices of the BLM in crafting the Plan of Development for the site. Patrick A. Shea assisted the collaboration with valuable advice and supported the collaboration's efforts. The people and the officials of Millard County, Utah have been a source of steadfast and warm support for our work which we greatly appreciate. We are indebted to the Millard County Road Department for their efforts to maintain and clear the roads which get us to our sites. We gratefully acknowledge the contribution from the technical staffs of our home institutions. An allocation of computer time from the Center for High Performance Computing at the University of Utah is gratefully acknowledged.

\section{References}

[1] Telescope Array collab., R. Abbasi et al., Astrophys. J. 858 (2018) 76, [1801.09784].

[2] Telescope Array collab., R. Abbasi et al., Phys. Rev. D 99 (2019) 022002, [1808.03680].

[3] Pierre Auger collab., A. Aab et al., JCAP 04 (2017) 038, [1612.07155].

[4] Pierre Auger collab., A. Aab et al., Phys. Rev. D 96 (2017) 122003, [1710.07249].

[5] A. Neronov and I. Vovk, Science 328 (2010) 73, [1006. 3504].

[6] M. Pshirkov, P. Tinyakov and F. Urban, Phys. Rev. Lett. 116 (2016) 191302, [1504. 06546].

[7] M. Haverkorn, in Magnetic Fields in Diffuse Media (A. Lazarian, E. M. de Gouveia Dal Pino and C. Melioli, eds.), vol. 407, p. 483, Jan., 2015, [1406.0283].

[8] M. S. Pshirkov et al., Astrophys. J. 738 (2011) 192, [1103.0814].

[9] R. Jansson and G. R. Farrar, Astrophys. J. 757 (2012) 14, [1204 . 3662].

[10] M. Y. Kuznetsov and P. G. Tinyakov, JCAP 04 (2021) 065, [2011. 11590].

[11] Telescope Array collab., T. Abu-Zayyad et al., NIM A689 (2013) 87, [1201.4964].

[12] H. Tokuno et al., NIM A676 (2012) 54, [1201.0002].

[13] Telescope Array, Pierre Auger collabs., A. Aab et al., Astrophys. J. 794 (2014) 172, [1409.3128].

[14] K. Cummins and M. J. Murphy, IEEE Trans. 51 (2009) 499.

[15] Telescope ArRay collab., R. U. Abbasi et al., MNRAS 492 (2020) 3984, [1904.00300].

[16] R. Aloisio et al., JCAP 11 (2017) 009, [1705. 03729].

[17] A. di Matteo and P. Tinyakov, MNRAS 476 (2018) 715, [1706. 02534].

[18] D. Ivanov, PoS ICRC2015 (2016) 349. 


\section{The Telescope Array Collaboration}

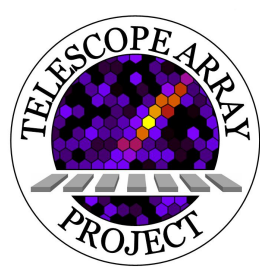

R.U. Abbasi ${ }^{1,2}$, T. Abu-Zayyad ${ }^{1,2}$, M. Allen ${ }^{2}$, Y. Arai ${ }^{3}, \quad$ R. Arimura ${ }^{3}$, E. Barcikowski², J.W. Belz ${ }^{2}$, D.R. Bergman' ${ }^{2}$, S.A. Blake ${ }^{2}$, I. Buckland² ${ }^{2}$, R. Cady ${ }^{2}$, B.G. Cheon $^{4}$, J. Chiba ${ }^{5}$, M. Chikawa ${ }^{6}$, T. Fujii ${ }^{7}$, K. Fujisue ${ }^{6}$, K. Fujita ${ }^{3}$, R. Fujiwara ${ }^{3}$, M. Fukushima ${ }^{6}$, R. Fukushima ${ }^{3}$, G. Furlich ${ }^{2}$, R. Gonzalez ${ }^{2}$, W. Hanlon ${ }^{2}$, M. Hayashi ${ }^{8}$, N. Hayashida ${ }^{9}$, K. Hibino ${ }^{9}$, R. Higuchi ${ }^{6}$, K. Honda ${ }^{10}$, D. Ikeda ${ }^{9}$, T. Inadomi ${ }^{11}$, N. Inoue ${ }^{12}$, T. Ishii ${ }^{10}$, H. Ito ${ }^{13}$, D. Ivanov ${ }^{2}$, H. Iwakura ${ }^{11}$, A. Iwasaki ${ }^{3}$, H.M. Jeong ${ }^{14}$, S. Jeong ${ }^{14}$, C.C.H. Jui ${ }^{2}$, K. Kadota ${ }^{15}$, F. Kakimoto ${ }^{9}$, O. Kalashev ${ }^{16}$, K. Kasahara ${ }^{17}$, S. Kasami ${ }^{18}$, H. Kawai ${ }^{19}$, S. Kawakami ${ }^{3}$, S. Kawana ${ }^{12}$, K. Kawata ${ }^{6}$, I. Kharuk ${ }^{16}$, E. Kido ${ }^{13}$, H.B. Kim ${ }^{4}$, J.H. Kim ${ }^{2}$, J.H. Kim ${ }^{2}$, M.H. Kim ${ }^{14}$, S.W. Kim ${ }^{14}$, Y. Kimura ${ }^{3}$, S. Kishigami ${ }^{3}$, Y. Kubota ${ }^{11}$, S. Kurisu ${ }^{11}$, V. Kuzmin ${ }^{16, a}$, M. Kuznetsov ${ }^{16,20}$, Y.J. Kwon ${ }^{21}$, K.H. Lee ${ }^{14}$, B. Lubsandorzhiev ${ }^{16}$, J.P. Lundquist ${ }^{2,22}$, K. Machida ${ }^{10}$, H. Matsumiya ${ }^{3}$, T. Matsuyama ${ }^{3}$, J.N. Matthews ${ }^{2}$,

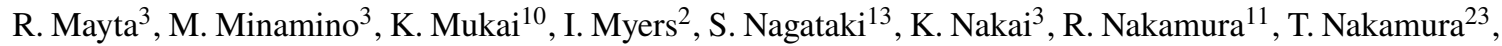
T. Nakamura ${ }^{11}$, Y. Nakamura ${ }^{11}$, A. Nakazawa ${ }^{11}$, E. Nishio ${ }^{18}$, T. Nonaka ${ }^{6}$, H. Oda ${ }^{3}$, S. Ogio ${ }^{3,24}$, M. Ohnishi ${ }^{6}$, H. Ohoka ${ }^{6}$, Y. Oku ${ }^{18}$, T. Okuda ${ }^{25}$, Y. Omura ${ }^{3}$, M. Ono ${ }^{13}$, R. Onogi ${ }^{3}$, A. Oshima ${ }^{3}$, S. Ozawa ${ }^{26}$, I.H. Park ${ }^{14}$, M. Potts ${ }^{2}$, M.S. Pshirkov ${ }^{16,27}$, J. Remington ${ }^{2}$, D.C. Rodriguez ${ }^{2}$, G.I. Rubtsov ${ }^{16}$, D. Ryu ${ }^{28}$, H. Sagawa ${ }^{6}$, R. Sahara ${ }^{3}$, Y. Saito ${ }^{11}$, N. Sakaki ${ }^{6}$, T. Sako ${ }^{6}$, N. Sakurai ${ }^{3}$, K. Sano ${ }^{11}$, K. Sato ${ }^{3}$, T. Seki ${ }^{11}$, K. Sekino ${ }^{6}$, P.D. Shah ${ }^{2}$, Y. Shibasaki ${ }^{11}$, F. Shibata ${ }^{10}$, N. Shibata ${ }^{18}$, T. Shibata ${ }^{6}$, H. Shimodaira ${ }^{6}$, B.K. Shin ${ }^{28}$, H.S. Shin ${ }^{6}$, D. Shinto ${ }^{18}$, J.D. Smith ${ }^{2}$, P. Sokolsky ${ }^{2}$, N. Sone ${ }^{11}$, B.T. Stokes ${ }^{2}$, T.A. Stroman ${ }^{2}$, Y. Takagi ${ }^{3}$, Y. Takahashi ${ }^{3}$,

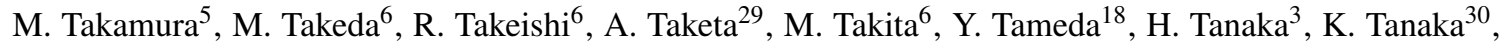
M. Tanaka ${ }^{31}$, Y. Tanoue ${ }^{3}$, S.B. Thomas ${ }^{2}$, G.B. Thomson ${ }^{2}$, P. Tinyakov ${ }^{16,20}$, I. Tkachev ${ }^{16}$, H. Tokuno $^{32}$, T. Tomida ${ }^{11}$, S. Troitsky ${ }^{16}$, R. Tsuda ${ }^{3}$, Y. Tsunesada ${ }^{3,24}$, Y. Uchihori ${ }^{33}$, S. Udo ${ }^{9}$, T. Uehama ${ }^{11}$, F. Urban ${ }^{34}$,

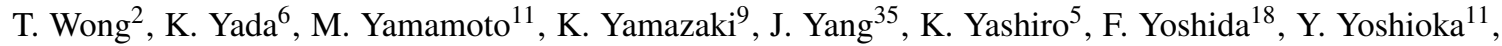
Y. Zhezher ${ }^{6,16}$, and Z. Zundel ${ }^{2}$

${ }^{1}$ Department of Physics, Loyola University Chicago, Chicago, Illinois, USA

2 The Graduate School of Science and Engineering, Saitama University, Saitama, Saitama, Japan

${ }^{3}$ High Energy Astrophysics Institute and Department of Physics and Astronomy, University of Utah, Salt Lake City, Utah, USA

${ }^{4}$ Graduate School of Science, Osaka City University, Osaka, Osaka, Japan

${ }^{5}$ Department of Physics and The Research Institute of Natural Science, Hanyang University, Seongdong-gu, Seoul, Korea

${ }^{6}$ Department of Physics, Tokyo University of Science, Noda, Chiba, Japan

${ }^{7}$ Institute for Cosmic Ray Research, University of Tokyo, Kashiwa, Chiba, Japan

${ }^{8}$ The Hakubi Center for Advanced Research and Graduate School of Science, Kyoto University, KitashirakawaOiwakecho, Sakyo-ku, Kyoto, Japan

${ }^{9}$ Kavli Institute for the Physics and Mathematics of the Universe (WPI), Todai Institutes for Advanced Study, University of Tokyo, Kashiwa, Chiba, Japan

${ }^{10}$ Information Engineering Graduate School of Science and Technology, Shinshu University, Nagano, Nagano, Japan

${ }^{11}$ Faculty of Engineering, Kanagawa University, Yokohama, Kanagawa, Japan

12 Interdisciplinary Graduate School of Medicine and Engineering, University of Yamanashi, Kofu, Yamanashi, Japan

13 Academic Assembly School of Science and Technology Institute of Engineering, Shinshu University, Nagano, Nagano, Japan

${ }^{14}$ Astrophysical Big Bang Laboratory, RIKEN, Wako, Saitama, Japan

15 Department of Physics, Sungkyunkwan University, Jang-an-gu, Suwon, Korea

${ }^{16}$ Department of Physics, Tokyo City University, Setagaya-ku, Tokyo, Japan

${ }^{17}$ Institute for Nuclear Research of the Russian Academy of Sciences, Moscow, Russia

${ }^{18}$ Faculty of Systems Engineering and Science, Shibaura Institute of Technology, Minato-ku, Tokyo, Japan 
${ }^{19}$ Department of Engineering Science, Faculty of Engineering, Osaka Electro-Communication University, Neyagawa-shi, Osaka, Japan

${ }^{20}$ Department of Physics, Chiba University, Chiba, Chiba, Japan

${ }^{21}$ Service de Physique Théorique, Université Libre de Bruxelles, Brussels, Belgium

22 Department of Physics, Yonsei University, Seodaemun-gu, Seoul, Korea

${ }^{23}$ Center for Astrophysics and Cosmology, University of Nova Gorica, Nova Gorica, Slovenia

${ }^{24}$ Faculty of Science, Kochi University, Kochi, Kochi, Japan

${ }^{25}$ Nambu Yoichiro Institute of Theoretical and Experimental Physics, Osaka City University, Osaka, Osaka, Japan

${ }^{26}$ Department of Physical Sciences, Ritsumeikan University, Kusatsu, Shiga, Japan

${ }^{27}$ Quantum ICT Advanced Development Center, National Institute for Information and Communications Technology, Koganei, Tokyo, Japan

${ }^{28}$ Sternberg Astronomical Institute, Moscow M.V. Lomonosov State University, Moscow, Russia

${ }^{29}$ Department of Physics, School of Natural Sciences, Ulsan National Institute of Science and Technology, UNIST-gil, Ulsan, Korea

${ }^{30}$ Earthquake Research Institute, University of Tokyo, Bunkyo-ku, Tokyo, Japan

${ }^{31}$ Graduate School of Information Sciences, Hiroshima City University, Hiroshima, Hiroshima, Japan

32 Institute of Particle and Nuclear Studies, KEK, Tsukuba, Ibaraki, Japan

${ }^{33}$ Graduate School of Science and Engineering, Tokyo Institute of Technology, Meguro, Tokyo, Japan

${ }^{34}$ Department of Research Planning and Promotion, Quantum Medical Science Directorate, National Institutes for Quantum and Radiological Science and Technology, Chiba, Chiba, Japan

35 CEICO, Institute of Physics, Czech Academy of Sciences, Prague, Czech Republic

${ }^{36}$ Department of Physics and Institute for the Early Universe, Ewha Womans University, Seodaaemun-gu, Seoul, Korea

${ }^{a}$ Deceased 